\title{
The PHF11 gene is not associated with asthma or asthma phenotypes in two independent populations
}

\author{
J McClenaghan, ${ }^{1}$ N M Warrington, ${ }^{1}$ E F Jamrozik, ${ }^{1} \mathrm{~J} \mathrm{Hui}{ }^{2,3}$ J P Beilby, ${ }^{2,3} \mathrm{~J}$ Hansen, ${ }^{4}$ \\ N H de Klerk, ${ }^{4}$ A L James, ${ }^{5,6}$ A W Musk, ${ }^{5,6}$ L J Palmer ${ }^{1,7,8}$
}

- Additional details are published online only at http:// thorax.bmi.com/content/vol64/ issue7

${ }^{1}$ Centre for Genetic

Epidemiology and Biostatistics, University of Western Australia, Nedlands, Australia; ${ }^{2}$ Clinical Biochemistry, PathWest Laboratory Medicine, Perth, Australia; ${ }^{3}$ School of Surgery and Pathology, University of

Western Australia, Perth, Australia; ${ }^{4}$ Division of

Biostatistics and Genetic Epidemiology, Telethon Institute for Child Health Research,

Centre for Child Health

Research, University of Western Australia, Perth, Australia;

${ }^{5}$ West Australian Sleep

Disorders Research Institute, Sir Charles Gardner Hospital,

Nedlands, Australia; ${ }^{6}$ School of Medicine and Pharmacology,

University of Western Australia, Crawley, Australia; ${ }^{7}$ Western Australian Institute for Medical Research and UWA Centre for Medical Research, University of Western Australia, Nedlands Australia; ${ }^{8}$ School of Population Health, University of Western Australia, Crawley, Australia

Correspondence to:

Professor L J Palmer, University of Western Australia, Nedlands, WA 6009, Australia; Iyle@ cyllene.uwa.edu.au

Received 11 October 2008 Accepted 26 March 2009 Published Online First 20 April 2009

\begin{abstract}
Background: Numerous areas of the human genome have previously been associated with asthma and asthma-related phenotypes, but few positive findings have been successfully replicated in independent populations. Initial studies have reported strong associations of variants in the plant homeodomain zinc finger protein 11 (PHF11) gene with serum IgE levels, asthma, airway hyper-responsiveness and childhood atopic dermatitis.

Objectives: To investigate the association of variants in the PHF11 gene with asthma and associated intermediate phenotypes in two independent Western Australian population-based samples.
\end{abstract}

Methods: A linkage-disequilibrium (LD)-tagging set of 20 single nucleotide polymorphisms (SNPs) was genotyped in PHF11 in two separate populations (total $n=2315$ ), a family-based twin study consisting of 230 families ( $n=992$ subjects) and a population-based nested casecontrol study consisting of 617 asthma cases and 706 controls. Information regarding asthma, respiratory physiology, atopy and environmental exposures was collected. Transmission disequilibrium tests, variance components models and generalised linear models were used to test for association between PHF11 SNPs and selected asthma outcomes (including longitudinal change in lung function).

Results: After correction for multiple testing, no statistically significant $(p<0.05)$ associations were found between PHF11 and either asthma or total serum lgE levels in either population. No statistically significant associations were found with any other asthma-associated phenotypes in either population.

Conclusions: Previously reported associations of PHF11 with asthma outcomes were not replicated in this study. This study suggests that PHF11 is unlikely to contain polymorphic loci that have a major impact on asthma susceptibility in our populations.

Asthma is a chronic inflammatory disease of the airways which results in variable airway obstruction and episodes of wheezing and breathlessness due to widespread narrowing of the airways in the lung. ${ }^{1}$ The prevalence of asthma in the general population of Australia varies depending on the definition of asthma diagnosis, but is generally accepted to be around $10-12 \%$ of adults and $12-15 \%$ of children in Australia, ${ }^{2}$ both of which are high by international standards. ${ }^{3}$

Asthma is a complex disease caused by intricate interactions among genetic and environmental influences. ${ }^{4}$ The heterogeneous and complex nature of the aetiology of asthma has made it difficult to pinpoint precisely the extent of attribution of individual genetic and environmental influences, and can be further complicated by incomplete penetrance, epistasis and phenotypic pleiotrophy. ${ }^{5}$ Difficulties in defining asthma, and the complexities of the pathogenic mechanisms underlying the disease, mean that intermediate asthma phenotypes are commonly used in genetic studies. ${ }^{6}$ Commonly measured intermediate phenotypes for asthma include raised total serum IgE levels, increased exhaled nitric oxide (eNO), decreased spirometric indices (eg, forced expiratory volume in $1 \mathrm{~s}, \mathrm{FEV}_{1}$ ) and airway hyper-responsiveness (AHR) to inhaled spasmogens. All of these phenotypes are highly heritable.?

There have been many genome-wide linkage scans for asthma, and several genes have been claimed as positionally cloned susceptibility loci. . $^{-12}$ The 13q14 region, in which the plant homeodomain zinc finger protein 11 (PHF11) gene resides, has demonstrated significant linkage $(p<0.05)$ with asthma, atopy and IgE levels in a number of studies. ${ }^{13-17}$ The genome-wide linkage scan of Zhang et al ${ }^{18}$ narrowed down this region of association with IgE levels to the PHF11 gene and two flanking genes on either side, with three SNPs identified as having independent associations with total serum IgE levels. In an independent study in children, Hersh et al ${ }^{19}$ reported marginal associations between PHF11 and asthma, as well as AHR, but found limited association with IgE levels.

The current interest in PHF11 as a potential candidate gene has arisen from positional approaches. Until recently there was no clearly defined biological mode of action for PHF11 in relation to asthma, although it has been suggested to play a role in chromatin-mediated transcriptional regulation and immunoregulation, especially of B lymphocytes. ${ }^{18}{ }^{20} \mathrm{~A}$ recent study by Clark et $a^{21}$ found a higher expression of PHF11 in Th1 cells than Th2 cells, as well as a marked increase in PHF11 expression in basophils and mature dendritic cells, although there was low expression in mast cells and eosinophils.

Given the complex nature of the aetiology of asthma and the frequent inability of secondary studies to replicate a positive result in a primary study, comprehensive replication of initial positive findings in gene-disease association studies has become critical for determining the validity of a candidate gene for a specific trait. ${ }^{22} 23$ To investigate the association of PHF11 with asthma phenotypes, we identified a linkage-disequilibrium (LD)-tagging set of SNPs in the gene and conducted genetic association studies in two independent comprehensively phenotyped Western Australian (WA) samples. The potential pathway(s) through 
which the PHF11 gene might act to influence asthma susceptibility remain unclear. Therefore, in addition to doctordiagnosed asthma, we also investigated a number of quantitative asthma-associated phenotypes.

\section{METHODS}

\section{Study populations}

Subjects were recruited from two independent Western Australian (WA) populations, the WA Twin Register (WATR) and the Busselton Health Study (BHS).

The WATR recruited families of multiple birth children to the WA Twin Child Health (WATCH) study; recruitment is described in detail elsewhere. ${ }^{24}$ A subset of WATCH study families whose twins were born between 1990 and 1995 was then recruited to the "WATCH for asthma" study. The 992 individuals from 230 different families provided the data described in this paper.

The BHS includes a series of seven cross-sectional population health surveys of adult residents in the Shire of Busselton since $1966 .{ }^{25}$ In 1994/5 a cross-sectional community follow-up study was undertaken of all available attendees of previous surveys. A case-control sample of unrelated subjects with $(n=617)$ and without $(n=706)$ doctor-diagnosed asthma was selected from the 1994/5 BHS cohort. For the analysis between longitudinal lung function and PHF11, data collected from the 1994/5 survey and up to seven previous surveys were used (mean number of surveys per subject 3.8 , range $2-7$ ).

\section{Physiological parameters}

WATCH and BHS subjects were assessed for a variety of demographic, physiological and genetic variables. ${ }^{25} 26$ Physiological parameters within both populations included the dichotomous variables asthma and atopy, eosinophil count and respiratory measures. WATCH subjects were also assessed for total serum IgE levels and eNO. Dose-response slopes were also collected from the BHS population only. Participants were considered asthmatic if they responded positively to the question: "Has your doctor ever told you that you had asthma/bronchial asthma?" Total IgE concentrations were calculated using the Immulite 2000 immunoassay analyser (Diagnostic Products Corporation, Los Angeles, USA). Atopy was determined by skin prick testing and was defined as the development of a weal $>3 \mathrm{~mm}$ to at least one allergen via a skin prick test.

\section{Selection of SNPs}

A set of LD-tagging SNPs was selected to tag PHF11 and 10000 base pairs in each direction $(n=20)$ using the HapMap Phase II database. $^{27}$ SNPs identified from HapMap were run through Haploview (V4.0). ${ }^{28}$ The minor allele frequency (MAF) was set at 0.05 , Hardy-Weinberg Equilibrium (HWE) p value was set at 0.05 and the $r^{2}$ threshold was set at 0.8. The three SNPs identified by Zhang et $a l^{18}$ as having an independent effect on total serum IgE levels were also genotyped.

\section{Genotyping}

SNPs were genotyped using tetra-primer amplification refractory mutation system polymerase chain reaction, generated by a primer design program accessible through the internet ${ }^{29}$ or by the TaqMan SNP Genotyping System (Applied Biosystems, ABI).

\section{Statistical analysis}

HWE was tested at each SNP locus through the use of a Markov chain random walk algorithm ${ }^{30}$ using unrelated individuals. Pairwise LD was analysed by a likelihood ratio test. ${ }^{31}$ Lewontin disequilibrium coefficient $\mathrm{D}^{\prime}$ and $\mathrm{r}^{2}$ were calculated for each pairwise comparison.

Generalised linear models (GLMs) were used to model the effects of multiple covariates and PHF11 genotypes on casecontrol status or normally distributed continuous outcomes in the BHS sample. The $\mathrm{FEV}_{1} / \mathrm{FVC}$ ratio, dose-response slope and eosinophil count were not normally distributed and were analysed using the Kruskal-Wallis test. Longitudinal genetic association analyses were conducted on $\mathrm{FEV}_{1}$ and $\mathrm{FEV}_{1} / \mathrm{FVC}$ ratio $^{25}$ using Linear Mixed Effects (LME) models. ${ }^{22}$ Transmission disequilibrium tests were used in the WATCH families to investigate the associations between qualitative traits and PHF11 genotypes. Within the WATCH analysis, if twins were monozygous, one twin was deleted from the analysis as monozygous twins are non-informative in a study of this nature. A general variance components model to partition observed phenotypic variance into genetic and non-genetic components was used to model the effects of multiple covariates and PHF11 genotypes on quantitative outcomes. ${ }^{33}$ All BHS analyses were undertaken in the SimHap V.1.0.0 program (http://www.genepi.org.au/simhap.html) or the $\mathrm{R}$ statistical language. ${ }^{34}$ WATCH analyses were undertaken using the QTDT v2.6.0 program. ${ }^{33}$

Sex, age, smoking status, height, weight and body mass index (BMI) were included as potential covariates in the multivariate models in both populations. Multiple testing was corrected for using the false discovery rate method. ${ }^{35}$ Statistical significance was defined at the 0.05 level.

\section{RESULTS}

\section{Population characteristics}

The characteristics of the subjects in the WATCH and BHS populations are shown in table 1. As expected, the WATCH population was significantly younger than the BHS population, reflecting the different recruitment criteria. Age-related parameters such as BMI were also significantly lower in the WATCH population.

A total of 20 SNPs were identified through HapMap as capturing common genetic variation within PHF11 with an $\mathrm{r}^{2}$ value $\geqslant 0.8 .^{28}$ These SNPs were genotyped and examined in the WATCH and BHS samples. The final set of 20 tag SNPs were found to provide 91\% coverage for all 58 common HapMapCEU SNPs within the target region (mean $\mathrm{r}^{2}=0.94$ ). Both populations included the three SNPs previously reported by Zhang et $a l^{18}$ as having independent effects on IgE levels. The distribution of genotypes was consistent with HWE $(p>0.05)$ in all SNPs within the WATCH population. Deviation from HWE was seen in only one SNP (rs7332573) within the BHS population $\left(p=2.2 \times 10^{-7}\right)$. This SNP was excluded from further analysis within the BHS and subsequently within WATCH.

The PHF11 SNPs genotyped were in only weak/modest LD (fig 1), reflecting the use of LD-tagging SNPs. Genotype and allele frequencies are given in table E1 in the online supplement.

Univariate analyses within the WATCH population did not show any statistically significant associations between any SNP and any phenotype when accounting for multiple testing (data not shown). Multivariate modelling within WATCH, including BMI, age, sex and smoking status as covariates, also failed to show any statistically significant association between the genotyped SNPs and any quantitative asthma phenotype, 
Table 1 Characteristics of subjects in the Western Australian Twin Child Health (WATCH) study and the Busselton Health Study (BHS)

\begin{tabular}{|c|c|c|c|c|c|}
\hline \multirow[b]{2}{*}{ Characteristic } & \multirow[b]{2}{*}{ WATCH } & \multicolumn{3}{|l|}{ BHS } & \multirow[b]{2}{*}{ p Value } \\
\hline & & All & Controls & Asthma cases & \\
\hline Number of participants & 992 & 1323 & 706 & 617 & - \\
\hline Mean (SD) age (years) & $22.4(15.4)$ & $53.8(17.3)$ & $57.7(16.0)$ & $49.5(17.6)$ & $<0.001^{*}$ \\
\hline Gender, no $(\%)$ & & & & & $0.11 \dagger$ \\
\hline Male & $458(46.2)$ & $757(57.2)$ & $395(55.9)$ & $362(58.7)$ & \\
\hline Female & $534(53.8)$ & $566(42.8)$ & $311(44.1)$ & $255(41.3)$ & \\
\hline Asthma, no (\%) & $200(20.2)$ & $617(46.6)$ & $0(0)$ & $617(100)$ & $<0.001 \dagger$ \\
\hline Atopy, no (\%) & $482(48.6)$ & $1093(82.6)$ & $570(80.7)$ & $523(84.8)$ & $<0.001 \dagger$ \\
\hline Eosinophil count $\left(10^{9} /\right) \S$ & $0.2(0.0-0.4)$ & $0.20(0.1-0.3)$ & $0.20(0.1-0.2)$ & $0.20(0.1-0.3)$ & $<0.001^{*}$ \\
\hline Mean (SD) FEV ${ }_{1}$ (I) & $2.76(0.87)$ & $2.81(0.98)$ & $2.82(0.94)$ & $2.80(1.04)$ & $<0.001^{*}$ \\
\hline Mean (SD) $\mathrm{FEV}_{1} / \mathrm{FVC}$ ratio & $0.84(0.08)$ & $0.75(0.09)$ & $0.77(0.07)$ & $0.74(0.10)$ & $<0.001^{*}$ \\
\hline Dose-response slope§ & - & $2.35(1.0-6.1)$ & $1.58(0.75-3.36)$ & $4.26(1.71-25.12)$ & - \\
\hline
\end{tabular}

${ }^{*}$ p Value using Mann-Whitney Cox test. †p Value calculated using $\chi^{2}$ test. †Considered as a binary outcome. Subjects who were non-smokers or stopped smoking at least 8 months previously were considered to be non-current smokers. Subjects who were current smokers were considered smokers. §Median (interquartile range). $q \mathrm{p}$ Value for WATCH vs all of BHS

BMI, body mass index; $\mathrm{FEV}_{1}$, forced expiratory volume in $1 \mathrm{~s}$; FVCD, forced vital capacity.

including total serum IgE levels, eosinophil count, eNO, $\mathrm{FEV}_{1}$ or the $\mathrm{FEV}_{1} / \mathrm{FVC}$ ratio (see table $\mathrm{E} 2$ in the online supplement). Multivariate outcomes of asthma for both WATCH and BHS can be seen in table 2 .

Univariate analyses within the BHS population suggested marginal associations between rs9568221 and $\mathrm{FEV}_{1}$ (dominant model: $p=0.02$ ) and rs9535259 and asthma (dominant model: $p=0.04$ ). Multivariate GLM analysis indicated that the association between rs9568221 and $\mathrm{FEV}_{1}$ remained statistically significant under a dominant model $(\beta=111.1$, $\mathrm{SE}=42.3, \mathrm{p}=0.01)$, although this result was not statistically significant once multiple testing was accounted for $(q=0.21)$. After adjusting for other covariates, the association between asthma and rs9535259 was no longer statistically significant (dominant model: odds ratio $0.81,95 \%$ CI 0.63 to 1.03 , $\mathrm{p}=0.09$ ). All multivariate data for the Busselton population, excluding asthma, are presented in table E3 in the online supplement.

A significant association was also observed between a longitudinal decline in the $\mathrm{FEV}_{1} / \mathrm{FVC}$ ratio and $\mathrm{rs} 9568222$ (dominant model: coefficient $=-0.0009$, $\mathrm{SE}=0.0004$, $\mathrm{p}=0.02)$. Again, this association became statistically non-

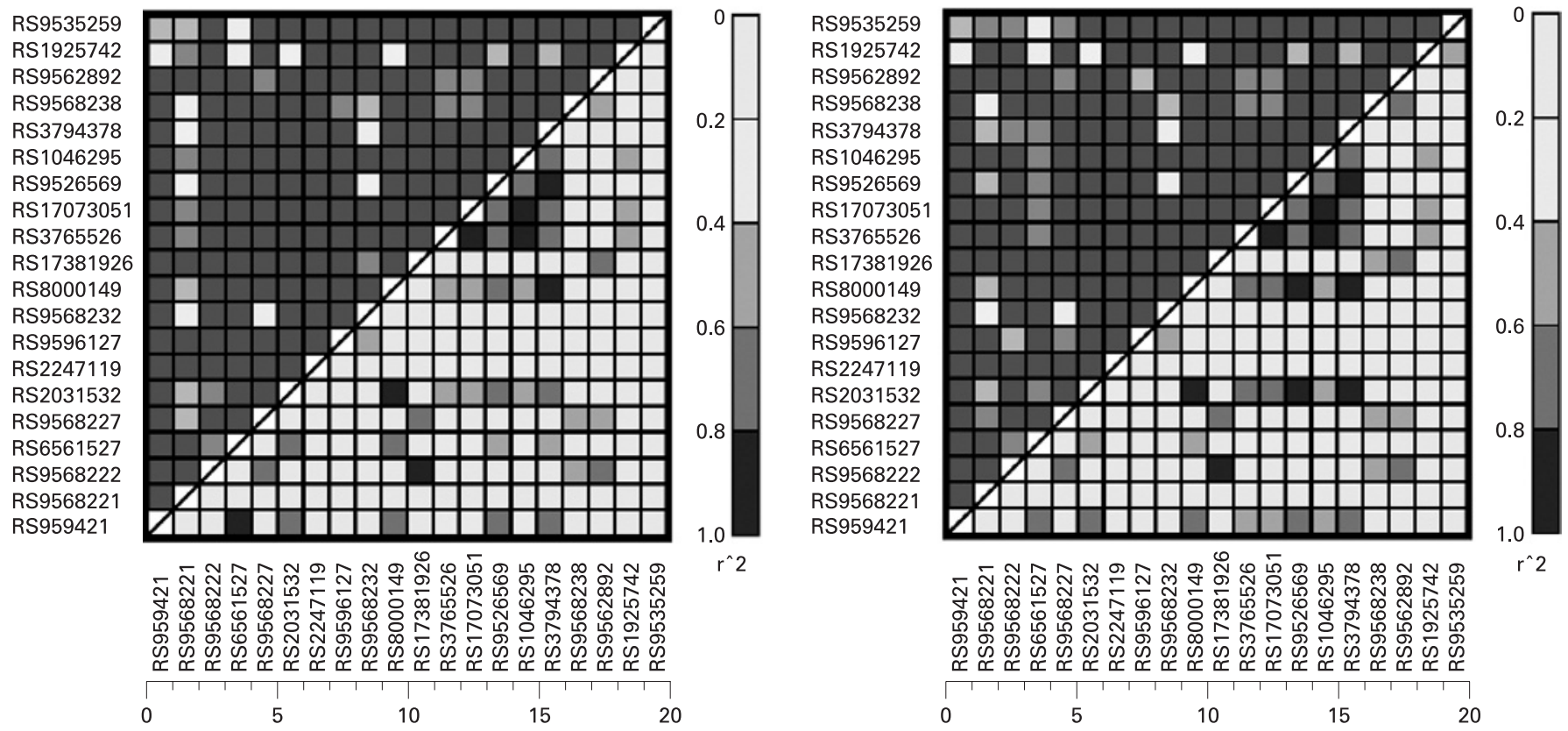

Figure 1 Comparison of linkage disequilibrium plots of PHF11-tagging SNPs genotyped in the Western Australian Twin Child Health (WATCH) study and the Busselton Health Study (BHS). 
Table 2 Multivariate genetic association with asthma in the Western Australian Twin Child Health (WATCH) study and the Busselton Health Study (BHS)

\begin{tabular}{|c|c|c|}
\hline \multirow[b]{2}{*}{ SNP } & Busselton & \multirow{2}{*}{$\begin{array}{l}\text { WATCH } \\
\text { p Value* }\end{array}$} \\
\hline & Genotype: coefficient $(95 \% \mathrm{CI})$, p value & \\
\hline \multirow[t]{2}{*}{ rs9568221 } & AG: $0.76(0.55$ to 1.05$), p=0.09$ & 0.34 \\
\hline & GG: $0.43(0.07$ to 2.50$), p=0.35$ & \\
\hline \multirow[t]{2}{*}{ rs9568222 } & AT: 1.35 (0.92 to 2.03$), p=0.14$ & $-\dagger$ \\
\hline & TT: $1.68(0.18$ to 2.79$), p=0.96$ & \\
\hline \multirow[t]{2}{*}{ rs6561527 } & AT: 0.89 (0.63 to 1.25$), p=0.53$ & 0.93 \\
\hline & TT: 1.70 (0.87 to 3.34$), p=0.11$ & \\
\hline rs9568227 & GA and $\mathrm{AA}: 1.26$ (0.87 to 1.84$), \mathrm{p}=0.21 \%$ & 0.88 \\
\hline \multirow[t]{2}{*}{ rs2031532 } & GA: $0.98(0.77$ to 1.25$), p=0.86$ & 0.87 \\
\hline & AA: $0.83(0.58$ to 1.20$), p=0.32$ & \\
\hline \multirow[t]{2}{*}{ rs2247119 } & CT: 0.99 (0.78 to 1.26$), p=0.98$ & 0.60 \\
\hline & CC: $1.11(0.74$ to 1.65$), p=0.62$ & \\
\hline rs9596127 & GT and TT: $1.12(0.70$ to 1.79$), p=0.62 \$$ & - \\
\hline \multirow[t]{2}{*}{ rs9568232 } & CT: 1.08 (0.76 to 1.52$), p=0.65$ & - \\
\hline & TT: $2.23(0.19$ to 25.2$), p=0.52$ & \\
\hline \multirow[t]{2}{*}{ rs8000149 } & TC: $1.013(0.79$ to 1.29$), p=0.91$ & 0.69 \\
\hline & CC: 0.82 (0.57 to 1.17$), p=0.29$ & \\
\hline \multirow[t]{2}{*}{ rs17381926 } & TC: $1.46(0.93$ to 2.28$), p=0.09$ & - \\
\hline & CC: 1.67 (0.97 to 2.84$), p=0.96$ & \\
\hline \multirow[t]{2}{*}{ rs3765526 } & CA: 1.02 (0.78 to 1.32$), p=0.87$ & 0.54 \\
\hline & AA: 0.93 (0.67 to 1.27$), p=0.64$ & \\
\hline \multirow[t]{2}{*}{ rs 17073051} & AG: $1.01(0.77$ to 1.31$), p=0.93$ & 0.46 \\
\hline & GG: $0.92(0.67$ to 1.27$), p=0.63$ & \\
\hline \multirow[t]{2}{*}{ rs9526569 } & TC: 0.99 (0.77 to 1.26$), p=0.94$ & 0.90 \\
\hline & CC: 0.83 (0.59 to 1.17$), p=0.30$ & \\
\hline \multirow[t]{2}{*}{ rs1046295 } & AG: $0.95(0.73$ to 1.24$), p=0.75$ & 0.58 \\
\hline & GG: $0.92(0.67$ to 1.26$), p=0.60$ & \\
\hline \multirow[t]{2}{*}{ rs3794378 } & AG: $1.07(0.74$ to 1.54$), p=0.69$ & 0.96 \\
\hline & GG: $1.22(0.72$ to 2.05$), p=0.44$ & \\
\hline \multirow[t]{2}{*}{ rs9568238 } & CT: $1.24(0.90$ to 1.70$), p=0.17$ & 0.51 \\
\hline & TT: $0.31(0.05$ to 1.66$), p=0.07$ & \\
\hline \multirow[t]{2}{*}{ rs9562892 } & CT: 1.28 (0.87 to 1.87$), p=0.19$ & 0.45 \\
\hline & TT: $0.18(0.01$ to 1.79$), p=0.14$ & \\
\hline \multirow[t]{2}{*}{ rs1925742 } & TC: 0.99 (0.79 to 1.26$), p=0.96$ & 0.21 \\
\hline & CC: 0.76 (0.50 to 1.13$), p=0.18$ & \\
\hline \multirow[t]{2}{*}{ rs9535259 } & GC: 0.82 (0.63 to 1.06 ), $p=0.13$ & 0.58 \\
\hline & CC: $0.72(0.39$ to 1.35$), p=0.32$ & \\
\hline
\end{tabular}

\footnotetext{
${ }^{*}$ Only $p$ values are shown here as transmission disequilibrium tests do not allow for estimations of odds ratios and $95 \%$ confidence intervals.

$\uparrow$ Not tested due to low numbers of informative families to run the transmission disequilibrium test.

$\dagger$ The number of participants with minor homozygous genotypes was too small to allow comparison under a co-dominant model and have been examined instead under a dominant model.
}

significant when multiple testing was taken into account $(q=0.10)$. No other SNP within PHF11 was significantly associated with the longitudinal lung function measures.

\section{DISCUSSION}

Our study was designed to select a set of SNPs capturing common genetic variation within the PHF11 gene, and to investigate a possible association between these selected variants and asthma-associated phenotypes in two well-characterised population-based samples. This study comprises a replication study in both child and adult populations of the associations reported by Zhang et $a l^{18}$ and Hersh et $a l^{19}$ with total serum IgE levels, asthma and AHR, and has examined the same SNPs identified as having an independent effect on IgE levels in the study by Zhang et al ${ }^{18}$ The current study is both relatively large and the only study, to the authors' knowledge, to investigate the association between PHF11 and the asthma-associated pheno- types of spirometric indices, eNO levels, eosinophil counts and longitudinal changes in lung function. We observed a statistically significant association between rs9568221 and $\mathrm{FEV}_{1}(\mathrm{p}=0.01)$ and rs9568222 and a decline in $\mathrm{FEV}_{1} / \mathrm{FVC}$ function $(\mathrm{p}=0.02)$ after adjustments for covariates. Both SNPs are located in introns within SETDB2 which were tagged to account for possible genetic effects either side of the PHF11 gene (see table E1 in the online supplement for SNP locations).

After accounting for multiple testing, no statistically significant association was found between any SNP within PHF11 and doctor-diagnosed asthma, atopy, eNO level, eosinophil count, $\mathrm{FEV}_{1}, \mathrm{FEV}_{1} / \mathrm{FVC}$ ratio, dose-response slope to methacholine or longitudinal change in lung function in either children or adults. In particular, there was no association between PHF11 and total serum IgE levels, the quantitative trait first used to map the gen ${ }^{18}$ and the reason that PHF11 is often quoted as a potential asthma gene.

The potential role of PHF11 in the pathogenesis of asthma is uncertain, but recent research is shedding light on the possible role of PHF11 and atopy. PHF11 encodes the gene NY-REN-34, originally identified in patients with renal cell carcinoma, and contains two plant homeodomain zinc finger proteins. Evidence so far suggests a role in protein binding and/or homodimerisation. ${ }^{18}$ Expression of PHF11 was found to be higher in Th1 cells than in Th2 cells, and it is theorised that PHF11 may act in concert with the NF- $\mathrm{kB}$ pathway as a transcriptional activator. ${ }^{21}$ In atopic individuals there tends to be an imbalance in Th1 and Th2 cells, favouring Th2. ${ }^{21}$ The evidence suggests that the PHF11 gene may regulate factors which encourage the formation of Th1 cells and that mutations within PHF11 will result in the preferential activation of Th2 cells and thus will be more common in individuals displaying atopic tendencies. Despite these links with the pathophysiology of atopy, PHF11 was identified as a potential asthma candidate gene through a positional approach rather than because of a known biological mode of function.

To date, one study has reported statistically significant associations between PHF11 and IgE serum levels and asthma, ${ }^{18}$ a subsequent study reported an association between PHF11 and asthma, ${ }^{19}$ and one study has reported an association between PHF11 and atopic dermatitis. ${ }^{36}$ The observed association between PHF11 and IgE, first reported by Zhang et al, ${ }^{18}$ was not replicated by Hersh et al. ${ }^{19}$ Hersh et al ${ }^{19}$ demonstrated an association with only one SNP, which displayed a statistically significant association to total serum IgE levels (rs9568232; $p=0.03$ in one study population only). The level of marginal $(0.05 \geqslant p \geqslant 0.03)$ replication demonstrated by Hersh et al was only noted at the gene level (ie, no single SNP associations were replicated across the two populations studied for PHF11). ${ }^{19} \mathrm{~A}$ difference in results may also be attributed to differences in phenotypic definition between the studies.

It is of interest to note that the population used by Zhang et $a l^{18}$ for the primary mapping of PHF11 and the two flanking genes came from 80 nuclear families recruited in 1992 from the town of Busselton, the same town from which participants in the BHS were drawn. The population was selected to be informative for atopy and had to include families with atopic and non-atopic individuals. To the best of our knowledge, there is no overlap between the population in our study (derived from the 1994/5 BHS follow-up study) and that of Zhang et al. ${ }^{18}$ For the current study, participants were derived from an unselected general population sample. In contrast, the sample of families from the 1992 study reported by Zhang et a ${ }^{18}$ were selected to 
Table 3 Association results of SNPs examined in this study which have been examined in previous populations

\begin{tabular}{|c|c|c|c|c|c|c|}
\hline SNP rs number & This study & Zhang et $\left.a\right|^{18}$ (IgE) & Zhang et al ${ }^{18}$ (asthma) & $\begin{array}{l}\text { Hersh et al }{ }^{19} \text { Costa } \\
\text { Rica (asthma) }\end{array}$ & $\begin{array}{l}\text { Hersh et al }{ }^{19} \\
\text { CAMP (asthma) }\end{array}$ & $\begin{array}{l}\text { Jang et } a f^{36} \text { (atopic } \\
\text { dermatitis) }\end{array}$ \\
\hline rs2031532 & $>0.05$ & 0.016 & - & $>0.05$ & $>0.05$ & $>0.05$ \\
\hline rs2247119 & $>0.05$ & 0.048 & - & $>0.05$ & $>0.05$ & 0.029 \\
\hline rs2274276 & $>0.05$ & 0.003 & - & $>0.05$ & $>0.05$ & $>0.05$ \\
\hline rs9526569 & $>0.05$ & 0.0005 & - & - & - & - \\
\hline rs1046295 & $>0.05$ & 0.001 & 0.017 & - & - & 0.007 \\
\hline
\end{tabular}

Only SNPs examined and found to be statistically significant in one or more studies have been included. A dash is indicative of the fact that the SNP was not examined in the population.

CAMP, Childhood Asthma Management Program (population of children with mild to moderate asthma and their parents).

be informative for asthma; this may in part account for the different results observed.

A summary of genetic association analysis of SNPs examined in the four studies published to date (including this study) is shown in table 3.

The results of association with atopic dermatitis have been included as asthma and atopic diseases may share similar genetic pathways. ${ }^{37}$ The SNP rs1046295 looks to be the most promising SNP as it has previously been significantly $(p<0.05)$ associated with asthma, ${ }^{18}$ IgE levels ${ }^{18}$ and atopic dermatitis ${ }^{36}$ in three separate populations. However, no statistically significant association was demonstrated in the current study.

Owing to the marked heterogeneity of the asthma phenotype, additional phenotypic parameters were analysed in our two populations and it was thus necessary to adjust for multiple testing. All the chosen phonotypes are related to asthma and may potentially allow further characterisation of genetic effects. Multiple testing in this study was adjusted for using the false discovery rate method and adjusts for the number of SNPs within each phenotype, thus the number of phenotypic end points examined will not affect the overall multiple adjustment. Multiple analyses were not taken into account by Hersh et al. ${ }^{19}$

Allele frequencies were very similar in the WATCH and BSH populations (see table E1 in the online supplement). Comparison of MAFs in our two populations and other reported populations ${ }^{18}{ }^{19}$ presents greater difficulty, as the SNPs used for analysis have not been consistent across studies.

A post hoc power calculation indicated that, assuming an $\alpha$ value of 0.05 under a dominant model, our study had $80 \%$ power to detect a true odds ratio of $\geqslant 1.46$ for asthma between cases and controls for an SNP with a MAF $\geqslant 5 \%$. The lowest MAF for an SNP in our study was 3.0\% (rs9596127). Assuming a MAF of $3.0 \%$, our study had $68 \%$ power to detect an odds ratio of 1.4 and $85 \%$ power to detect an odds ratio of 1.5 . In comparison, Hersh et al reported $80 \%$ power to detect an odds ratio of 1.66 for a MAF of $\geqslant 10 \%$ within the Costa Rica population and an ability to detect a lower odds ratio (not specified) within the Childhood Asthma Management Program (CAMP) population. ${ }^{19}$ Our study therefore suggests that $P H F 11$ is unlikely to contain a major locus modulating asthma risk in these study populations of predominantly Western European origin.

Failure of secondary studies to replicate positive results of an initial study is not uncommon in the genetic association literature, and there are numerous reasons cited as to why replication may fail. 2233839 These include (but are not limited to) small sample sizes resulting in insufficient power to detect minor genetic influences, poor study design, the analysis of different variants in replication studies, publication bias, known or unknown differences between populations, the heterogeneous nature of complex diseases and, more specifically, the differing definitions of what constitutes a positive outcome of asthma and atopy. ${ }^{22} 40$ There are estimates that, of the total number of studies which initially report a positive finding, $70-95 \%$ cannot be confirmed in subsequent studies. ${ }^{41}$ This highlights the need for careful confirmatory studies in independent populations of sufficient sample size. Positive publication bias is also likely to be a factor in the reporting of candidate gene studies $^{22}$ and may be a factor in the reported positive association with PHF11. Associations between variations in susceptibility genes and asthma or asthma phenotypes will be difficult to identify as each individual gene will probably be associated with a modest effect on phenotype. ${ }^{42}$

This study fulfils the major criteria for a meaningful replication of genetic association studies ${ }^{23}$ and has the following strengths: (1) PHF11 was comprehensively tagged to capture common genetic variation across the gene; (2) this is a large internally replicated genetic association study of samples totalling 2315 subjects of European ancestry genotyped for 20 PHF11 SNPs; and (3) the subjects were homogeneous and well characterised. Asthma was defined similarly in the current study and that of Zhang et al (questionnaire-based). However, the criteria used by Hersh et $a l^{19}$ to define asthma was more comprehensive and included methacholine hyper-responsivness (hyper-responsivness was defined as a provocative concentration of methacholine causing a $20 \%$ fall in $\mathrm{FEV}_{1}$ of $\left.\leqslant 12.5 \mathrm{mg} / \mathrm{ml}\right) .{ }^{19}$

In conclusion, this replication study suggests that PHF11 is not a major susceptibility locus for asthma in our populations. Further attempted replication in different populations will be important, especially in populations of non-European ethnicity. The current study does not preclude the possibility that PHF11 variants interact with environmental factors or act epistatically with genetic variation at other loci to affect disease or trait outcomes.

Acknowledgements: The authors thank the people of the Busselton community for their participation in this study and the participants involved in the WATCH for Asthma study; the Busselton Population Medical Research Foundation and the many colleagues who assisted in the collection of these data; the generous support for the 1994/5 follow-up study from Healthway, Western Australia. The Busselton population studies are supported by The Great Wine Estates of the Margaret River region of Western Australia.

Funding: The authors acknowledge the assistance of the Western Australian DNA Bank (NHMRC Enabling Facility) with DNA samples for this study and the support provided by the Western Australian Genetic Epidemiology Resource (NHMRC Enabling Facility). They also gratefully acknowledge the support of the WA Health Promotion Foundation (Healthway) for providing the initial funds, allowing the establishment of the WATR, the Wind-Over-Water Foundation and the Australian National Health and Medical Research Council (NHMRC) for providing ongoing funding.

Competing interests: None. 


\section{REFERENCES}

1. Balkissoon R. Asthma overview. Primary Care: Clinics in Office Practice 2008:35:41-60.

2. Australian Centre for Asthma Monitoring. Asthma in Australia: findings from the 2004-5 National Health Survey. Cat. No. ACM 10. Canberra: Australian Institute of Health and Welfare, 2007.

3. Eder W, Ege MJ, von Mutius E. The asthma epidemic. N Engl J Med 2006;355:2226-35

4. Vercelli D. Discovering susceptibility genes for asthma and allergy. Nat Rev Immunol 2008;8:169-82.

5. Bleecker E, Postma D, Meyers D. Genetic susceptibility to asthma in a changing environment. Ciba Found Symp 1997;206(90-99).

6. Busse W, Lemanske R. Asthma. N Engl J Med 2001;344:350-62.

7. Palmer L, Cookson W. Genomic approaches to understanding asthma. Genome Res 2000;10:1280-7.

8. Ober C, Tsalenko A, Parry R, et al. A second-generation genomewide screen for asthma: susceptibility alleles in a founder population. Am J Hum Genet 2000;67:1154-62.

9. Dizier M, Besse-Schmittler C, Guilloud-Bataille M, et al. Genome screen for asthma and related phenotypes in the French EGEA study. Am J Respir Crit Care Med 2000;162:1812-8.

10. Laitinen T, Daly M, Rioux J, et al. A susceptibility locus for asthma-related traits on chromosome 7 revealed by genome-wide scan in a founder population. Nat Genet 2001;28:87-91.

11. Wjst M, Fischer G, Immervoll T, et al. A genome-wide search for linkage to asthma. Genomics 1999;58:1-8.

12. Collaborative Study on the Genetics of Asthma (CSGA). A genome-wide search for asthma susceptibility loci in ethnically diverse populations. Nat Genet 1997;15:389-92.

13. Daniels S, Bhattacharrya $S$, James $A$, et al. A genome-wide search for quantitative trait loci underlying asthma. Nature 1996;383:247-50.

14. Dizier MH, Besse-Schmittler C, Guilloud-Bataille M, et al. Genome screen for asthma and related phenotypes in the French EGEA study. Am J Respir Crit Care Med 2000:162:1812-5.

15. Hizawa N, Freidhoff LR, Chiu YF, et al. Genetic regulation of Dermatophagoides pteronyssinus-specific lgE responsiveness: a genome-wide multipoint linkage analysis in families recruited through two asthmatic sibs. J Allergy Clin Immunol 1998;102:436-42.

16. Xu J, Postma D, Howard T, et al. Major genes regulating total serum immunoglobulin E levels in families with asthma. Am J Hum Genet 2000;67:1163-73.

17. Ober C, Cox N, Abney M, et al. Genome-wide search for asthma susceptibility loci in a founder population: the collaborative study on the genetics of asthma. Hum $\mathrm{Mol}$ Genet 1998; 7:1393-8.

18. Zhang $\mathbf{Y}$, Leaves $\mathrm{N}$, Anderson $\mathrm{G}$, et al. Positional cloning of a quantitative trait locus on chromosome 13q14 that influences immunoglobulin E levels and asthma. Nat Genet 2003;34:181-6.

19. Hersh C, Raby BA, Soto-Quirós ME, et al. Comprehensive testing of positionally cloned asthma genes in two populations. Am J Respir Crit Care Med 2007;176:849-57.

20. Aasland R, Gibson T, Stewart A. The PHD finger: implications for chromatinmediated transcriptional regulation. Trends Biochem Sci 1995;20:56-9.
21. Clarke E, Rahman N, Page N, et al. Functional characterization of the atopyassociated gene PHF11. J Allergy Clin Immunol 2008;121:1148-54.

22. Ioannidis J, Ntzani E, Trikalinos T, et al. Replication validity of genetic association studies. Nat Genet 2001;29:306-9.

23. Chanock S, Manolio T, Boehnke M, et al. Replicating genotype-phenotype associations. Nature 2007; 447:655-9.

24. Hansen J, Alessandri P, Croft M, et al. The Western Australian Register of childhood multiples: effect of questionnaire design and follow-up protocol on response rates and representativeness. Twin Res 2004:7:149-61.

25. James A, Palmer L, Kicic E, et al. Decline in lung function in the Busselton Health Study: the effects of asthma and cigarette smoking. Am J Respir Crit Care Med 2005;171:109-14.

26. Hansen J, De Klerk N, Croft M, et al. The Western Australian Twin Child Health (WATCH) study: work in progress. Aust Epidemiol 2000;7:16-20.

27. Thorisson G, Smith A, Krishnan L, et al. A user's guide to the International HapMap project web site. 2005. http://www.hapmap.org/downloads/presentations/ users_guide_to_hapmappdf.

28. Barrett J, Fry $\bar{B}$, Maller J, et al. Haploview: analysis and visualization of $L D$ and haplotype maps. Bioinformatics 2005;21:263-5.

29. Ye S, Dhillon N, Ke X, et al. Tetra-primer ARMS-PCR. 2001. Updated 4 Nov 2006 http://cedar.genetics.soton.ac.uk/public html/primer1.html.

30. Guo SSW, Thompson EEA. Performing the exact test of Hardy-Weinberg proportion for multiple alleles. Biometrics 1992;48:361-72.

31. Slatkin $\mathbf{M}$, Excoffier L. Testing for linkage disequilibrium in genotypic data using the expectation-maximization algorithm. Heredity 1996;76:377-83.

32. Laird N, Ware J. Random-effects models for longitudinal data. Biometrics 1982;38:963-74.

33. Abecasis G, Cardon L, Cookson W. A general test of association for quantitative traits in nuclear families. Am J Hum Genet 2000;66:279-92.

34. Ihaka R, Gentleman R. A language for data analysis and graphics. J Comput Graph Stat 1996:5:299-314.

35. Benjamini Y, Yekutieli D. The control of the false discovery rate in multiple testing under dependency. Ann Stat 2001;29:1165-88

36. Jang N, Stewart G, Jones G. Polymorphisms within the PHF11 gene at chromosome $13 q 14$ are associated with childhood atopic dermatitis. Gene Immun 2005;6:262-4.

37. Palmer LJ, Burton PR, James AL, et al. Familial aggregation and heritability of asthma-associated quantitative traits in a population-based sample of nuclear families. Eur J Hum Genet 2000;8:853-60.

38. Chanock SJ, Manolio T, Boehnke M, et al. Replicating genotype-phenotype associations. Nature 2007; 447:655-60.

39. Davey Smith G, Ebrahim S, Lewis S, et al. Genetic epidemiology and public health: hope, hype and future prospects. Lancet 2005;366:1484-98.

40. Altmüller J, Palmer LJ, Fischer G, et al. Genome-wide scans of complex human diseases: true linkage is hard to find. Am J Hum Genet 2001;69:936-50.

41. Herrington D. Eliminating the improbable: Sherlock Holmes and standards of evidence in the genomic age. Circulation 2005:112:2081-4.

42. Gray I, Campbell D, Spurr N. Single nucleotide polymorphisms as tools in human genetics. Hum Mol Genet 2000;9:2403-8. 